\title{
Dead magnetic layers at the interface: Moment quenching through hybridization and frustration
}

\author{
Sebastian Meyer $\odot,{ }^{1}$ Martin Schmitt, ${ }^{2}$ Matthias Vogt, ${ }^{2}$ Matthias Bode $\odot,{ }^{2,3}$ and Stefan Heinze ${ }^{1}$ \\ ${ }^{1}$ Institut für Theoretische Physik und Astrophysik, Christian-Albrechts-Universität zu Kiel, Leibnizstrasse 15, 24098 Kiel, Germany \\ ${ }^{2}$ Physikalisches Institut, Experimentelle Physik II, Universität Würzburg, Am Hubland, 97074 Würzburg, Germany \\ ${ }^{3}$ Wilhelm Conrad Röntgen-Center for Complex Material Systems (RCCM), Universität Würzburg, Am Hubland, 97074 Würzburg, Germany
}

(Received 27 September 2019; revised manuscript received 2 March 2020; accepted 9 March 2020; published 23 March 2020)

\begin{abstract}
Using spin-polarized scanning tunneling microscopy (STM) we observe a two-dimensional $c(2 \times 2)$ antiferromagnetic state in a pseudomorphic Mn double layer on the W(001) surface. First-principles calculations confirm the antiferromagnetic ground state. Surprisingly, only the surface Mn layer possesses a magnetic moment while the moments of interface Mn atoms vanish. Calculated STM and spin-polarized STM images as well as local vacuum density of states are in good agreement with experiments and provide strong evidence of a dead magnetic interface layer.
\end{abstract}

DOI: 10.1103/PhysRevResearch.2.012075

Introduction. Antiferromagnetic materials are currently receiving a lot of attention due to the prospect of using them in novel magnetic data storage, logic, or optospintronic devices [1]. Due to faster spin dynamics, insensitivity to magnetic fields, and vanishing stray fields [2,3], antiferromagnetic (AFM) spintronic devices are expected to exhibit superior properties as compared to their ferromagnetic (FM) counterparts [4]. Relativistic and topological phenomena play an essential role in this growing field [5]. For example, the discovery of electrical switching of antiferromagnets [6] based on relativistic spin transport phenomena [7] has been an essential step towards potential applications.

$\mathrm{Mn}$ is a particularly intriguing AFM material with a diverse interplay between structural and magnetic properties [8]. It exists in four allotropes, where the low-temperature phases $\alpha$ - and $\beta$-Mn exhibit very large, crystallographically highly complex unit cells. More simple cubic phases, i.e., facecentered-cubic (fcc) $\gamma$-Mn and body-centered-cubic (bcc) $\delta$ $\mathrm{Mn}$, are stable only at temperatures close to the melting point, such that they cannot order magnetically in the bulk. A route for stabilizing $\gamma$ - and $\delta$-Mn is by epitaxial growth on suitable substrates [9-15]. Early calculations for $\delta$-Mn predicted a lattice constant-dependent low-spin (LS) $\rightarrow$ high-spin (HS) transition [16,17], potentially due to a type-III phase transition [18-20], which may be experimentally accessible by tuning of the lattice constant through strain.

In this context, (110) and (001) surfaces of W are promising bcc substrates, as the growth of $\mathrm{Mn}$ proceeds pseudomorphic up to a few atomic layers (ALs) [9,11], resulting in considerable tensile strain. Furthermore, electronic hybridization at the W-Mn interface leads to enhanced magnetic

Published by the American Physical Society under the terms of the Creative Commons Attribution 4.0 International license. Further distribution of this work must maintain attribution to the author $(s)$ and the published article's title, journal citation, and DOI. properties. For example, the exchange interaction of a $\mathrm{Mn}$ monolayer (ML) on $\mathrm{W}(110)$ is in general AFM [21], but the Dzyaloshinskii-Moriya interaction (DMI) enforces a cycloidal spin spiral [22]. The exchange interaction of the Mn double layer (DL) on W(110) also prefers an AFM order, but a conical spin spiral ground state is induced by the concerted action of DMI and higher-order exchange interactions [23]. In contrast, the nearest-neighbor exchange interaction for a Mn ML on W(001) is FM due to hybridization and the DMI induces a spin spiral state [24]. A density functional theory (DFT) study on the magnetic ground state was performed for up to three ALs of Mn/W(001) [25]. For a Mn DL on W(001), a FM ground state has been predicted, but experimental studies have not been reported.

Here, we show that the surface of a Mn DL on W(001) exhibits a two-dimensional $c(2 \times 2)$ AFM ground state. Scanning tunneling microscopy (STM) measurements confirm the pseudomorphic growth of the Mn DL and spin-polarized (SP)STM images of the surface atomic-scale magnetic structure in real space. DFT calculations performed using the fullpotential linearized augmented plane-wave (FLAPW) method confirm the surface $c(2 \times 2)$ AFM ground state. Surprisingly, the calculations also reveal that the magnetic moments of the interface Mn layer are quenched. It is a truly "dead magnetic layer" (DML) where the magnetic moment vanishes completely. In the past the experimental observation of DMLs was frequently claimed (see, e.g., Refs. [26-28] or Ref. [29] for a review) and usually attributed to $d$-state hybridization between the film and substrate [30,31]. Later studies revealed, however, that the magnetic moments in these layers were either vastly reduced due to oxidation at the surface or interface [32] or compensated by AFM order $[29,33]$.

Our comparison of the vacuum local density of states (LDOS) with STM spectra and calculated (SP-)STM images with experimental data verifies the $c(2 \times 2)$ AFM ground state and provides strong evidence for the dead magnetic $\mathrm{Mn}$ interface layer. The $\mathrm{Mn}$ atoms of this interface layer can be 

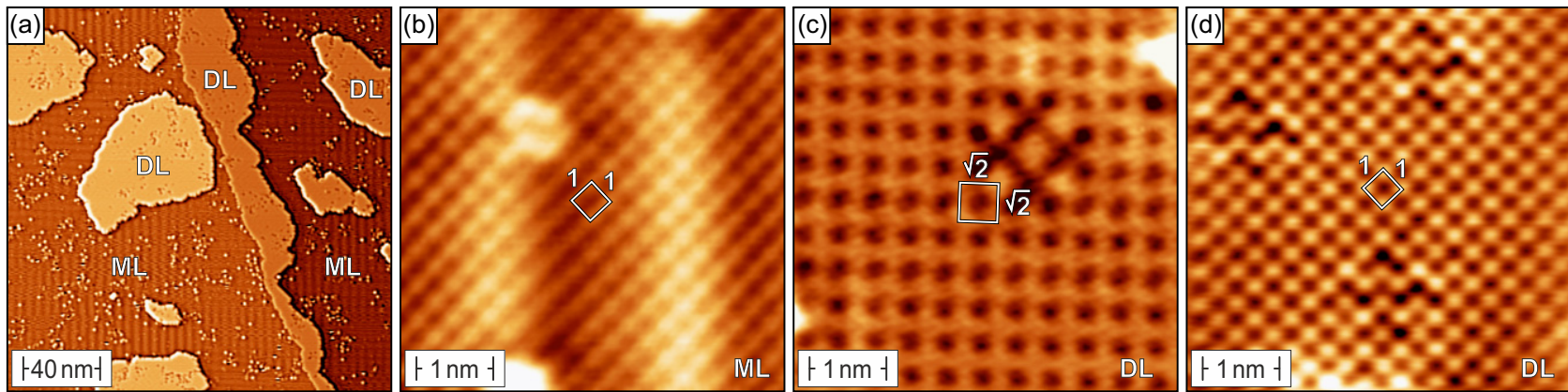

FIG. 1. (a) Overview scan of $1.3 \mathrm{AL} \mathrm{Mn/W(001)} \mathrm{measured} \mathrm{with} \mathrm{a} \mathrm{magnetic} \mathrm{Fe/W} \mathrm{tip.} \mathrm{(b)} \mathrm{Characteristic} \mathrm{magnetic} \mathrm{stripe} \mathrm{pattern} \mathrm{of} \mathrm{the}$ atomically resolved Mn ML. (c) Magnetic resolution and (d) spin-averaged data measured on top of a Mn DL island. The enlarged $(\sqrt{2} \times \sqrt{2})$ magnetic unit cell indicates AFM order. The colored arrows illustrate the magnetization directions of tip and sample, respectively. Scan parameters: (a) $U=1 \mathrm{~V}, I=1 \mathrm{nA}$; (b) $U=10 \mathrm{mV}, I=20 \mathrm{nA}$; (c) $U=10 \mathrm{mV}, I=3 \mathrm{nA}$; (d) $U=10 \mathrm{mV}, I=5 \mathrm{nA}$.

observed directly in non-spin-polarized STM images using SP-STM difference images with a reversed tip magnetization. Such subsurface imaging of buried atoms has been reported before for nonmagnetic metallic surfaces [34,35].

Experimental procedures. STM experiments were performed in a two-chamber ultrahigh vacuum (UHV) system with a base pressure $p \leqslant 5 \times 10^{-11}$ mbar. Clean $\mathrm{W}(001)$ was prepared by annealing cycles in an oxygen atmosphere followed by a high-temperature annealing without oxygen [36]. $\mathrm{Mn}$ was deposited from a $\mathrm{W}$ crucible with a commercial $e$-beam evaporator onto the substrate held at room temperature. After deposition the Mn films were annealed at $T \approx 520 \mathrm{~K}$, immediately followed by their transfer into a home-built low-temperature STM with an operation temperature of $T \approx 5.5 \mathrm{~K}$. Measurements were performed with electrochemically etched polycrystalline W tips. For SP-STM the $\mathrm{W}$ tips were flashed by electron bombardment and coated with Fe or Cr. The differential conductance $d I / d U$ was measured by adding a modulation voltage $U_{\text {mod }}=10 \mathrm{mV}$ to the sample bias $U$ and recording the resulting variation of the tunneling current $I$ by means of the lock-in technique.

Results. Figure 1(a) shows an overview constant-current SP-STM image of a 1.3 AL Mn film on W(001) measured with an Fe-coated tip. Whereas the surfaces of the islands and of a narrow stripe along a step edge are very smooth and only show a few pointlike depressions, the surrounding surface exhibits a stripe pattern which is characteristic for the pseudomorphic Mn ML on W(001) [24]. In agreement with literature [24], we find a periodicity of $(2.3 \pm 0.2) \mathrm{nm}$. Figure 1(b) shows an atomic-resolution SP-STM image of the Mn ML. The quadratic $(1 \times 1)$ structural unit cell (white square) is superimposed by a longer wavelength modulation caused by spin-polarized tunneling into a spin spiral that propagates along the substrate's $\langle 110\rangle$ directions [24].

Figure 1(c) presents atomic-scale SP-STM data measured on a DL island. We recognize a $(\sqrt{2} \times \sqrt{2})$ superstructure which is rotated by $45^{\circ}$ with respect to the atomic lattice. A qualitatively similar magnetic superstructure was observed for the Fe ML on W(001) [37]. We would like to note, however, that a detailed inspection of the magnetic contrast reveals some striking differences. Whereas the up and down magnetized Fe atoms of the AFM ML on W(001) appeared as protrusions and depressions, respectively, resulting in a distinctive checkerboard pattern with bright and dark "squares" in color-coded STM images [37], the Mn DL exhibits holes which are surrounded by a grid of linear elevations. As we will discuss below, this difference can be assigned to its unusual interfacial electronic and magnetic properties. To verify the magnetic origin of this enlarged unit cell we also scanned the Mn DL on W(001) with nonmagnetic $\mathrm{W}$ tips. A representative result of these spin-averaging experiments is shown in Fig. 1(d). The measured periodicity of $(312 \pm 30) \mathrm{pm}$ of the $(1 \times 1)$ structural unit cell confirms that $\mathrm{Mn}$ growth is pseudomorphic, i.e., with a bcc crystal structure, on $\mathrm{W}(001)$.

In order to understand the origin of these experimental observations we conducted DFT calculations based on the FLAPW method as implemented in the FLEUR code [38]. Structural relaxations have been performed within the generalized gradient approximation (GGA) [39] using a symmetric slab with a DL of Mn on both sides of a nine-layer W(001) substrate with an experimental W lattice constant of 5.981 a.u. The muffin tin radii of $\mathrm{Mn}$ are chosen to be 2.25 a.u., and 2.50 a.u. are used for $\mathrm{W}$. The $\mathrm{W} 5 p$ semicore states are described by local $p$ orbitals which are added to the basis set. We used an energy cutoff of $k_{\max }=4.0$ a.u. ${ }^{-1}$ and the $k$-point mesh consists of $289 k$ points in $1 / 4$ of the Brillouin zone (BZ). For relaxations, the forces were converged to less than $10^{-5}$ hartree/a.u. [40].

We have first considered three collinear magnetic structures: the ferromagnetic (FM), the layered antiferromagnetic (LAFM, i.e., two oppositely magnetized FM layers), and the $c(2 \times 2)$ AFM state. Table I shows the obtained relaxed interlayer distances and magnetic moments for all states [41]. We

TABLE I. Relaxed interlayer distances $d$ in atomic units and magnetic moments $m$ in $\mu_{\mathrm{B}}$ of the topmost three layers in the ferromagnetic (FM), layered antiferromagnetic (LAFM), and the $c(2 \times 2)$ antiferromagnetic $(\mathrm{AFM})$ state obtained in GGA.

\begin{tabular}{lcrrrrc}
\hline \hline & $d_{\mathrm{FM}}$ & $m_{\mathrm{FM}}$ & $d_{\mathrm{LAFM}}$ & $m_{\mathrm{LAFM}}$ & $d_{\mathrm{AFM}}$ & $m_{\mathrm{AFM}}$ \\
\hline $\mathrm{Mn}(\mathrm{S})$ & 2.80 & 3.81 & 2.53 & -3.70 & 2.74 & \pm 3.70 \\
$\mathrm{Mn}(\mathrm{I})$ & 2.46 & 1.18 & 2.77 & 2.38 & 2.50 & 0.0 \\
$\mathrm{~W}$ & 3.10 & -0.19 & 3.00 & -0.25 & 3.10 & \pm 0.03 \\
\hline \hline
\end{tabular}




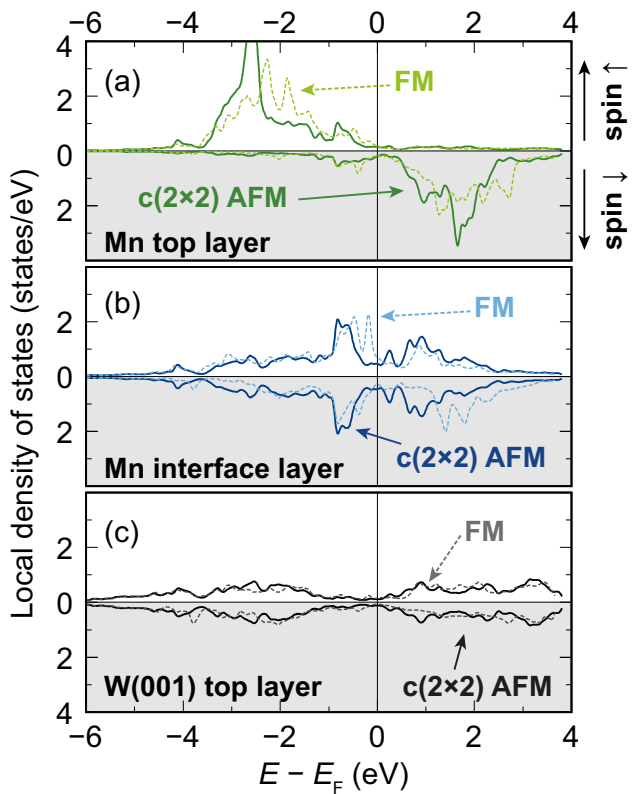

FIG. 2. Spin-resolved local density of states (LDOS) of the Mn double layer on $\mathrm{W}(001)$ in the ferromagnetic (FM, dashed lines) and $c(2 \times 2)$ antiferromagnetic (AFM) state (solid lines). (a) Mn surface layer, (b) Mn interface layer, and (c) W surface layer.

find that the $c(2 \times 2)$ AFM state is the lowest in total energy. The FM state is by $45.6 \mathrm{meV} / \mathrm{Mn}$ atoms higher in energy and the LAFM state by $91.9 \mathrm{meV} / \mathrm{Mn}$ atoms. Unexpectedly, the magnetic moment of the Mn interface layer vanishes in the $c(2 \times 2)$ AFM state while the Mn surface layer moments are of a similar magnitude in all three magnetic configurations. Even in the FM state the Mn interface layer carries only about $1.2 \mu_{\mathrm{B}}$, which is quite low for Mn with its half-filled $3 d$ band. The reduced magnetic moment can be related to the small interlayer distance between the Mn interface and the surface $\mathrm{W}$ layer. In both the FM and the $c(2 \times 2) \mathrm{AFM}$ state, the distance is much lower than in the LAFM state which exhibits a significantly higher interfacial moment of about $2.4 \mu_{\mathrm{B}}$.

We have confirmed the $c(2 \times 2)$ AFM ground state with vanishing magnetic moment for the Mn interface layer by performing spin spiral calculations in which we include noncollinear magnetic states and probe a large part of the magnetic configuration space [36]. Upon including spin-orbit coupling in these calculations, we find that compared to other Mn-W systems [21,23,24], the DMI is too weak in the Mn DL on $\mathrm{W}(001)$ to affect the collinear AFM state [36].

The LDOS displayed in Fig. 2 for the FM and the $c(2 \times 2)$ AFM state shows that the small interlayer distance leads to a strong hybridization between the interfacial $\mathrm{Mn}$ and the topmost W layer [37,42]. As expected, in both configurations the Mn top layer [Fig. 2(a)] exhibits a large exchange splitting between majority and minority $3 d$ bands, in good correspondence to the magnetic moment of $\approx 3.8 \mu_{\mathrm{B}}$. In contrast, the LDOS of the Mn interface layer is distinctively different [Fig. 2(b)]. For the FM state a small exchange splitting on the order of about $1 \mathrm{eV}$ remains, but the LDOS has become much broader due to hybridization with the $\mathrm{W}$ top layer.

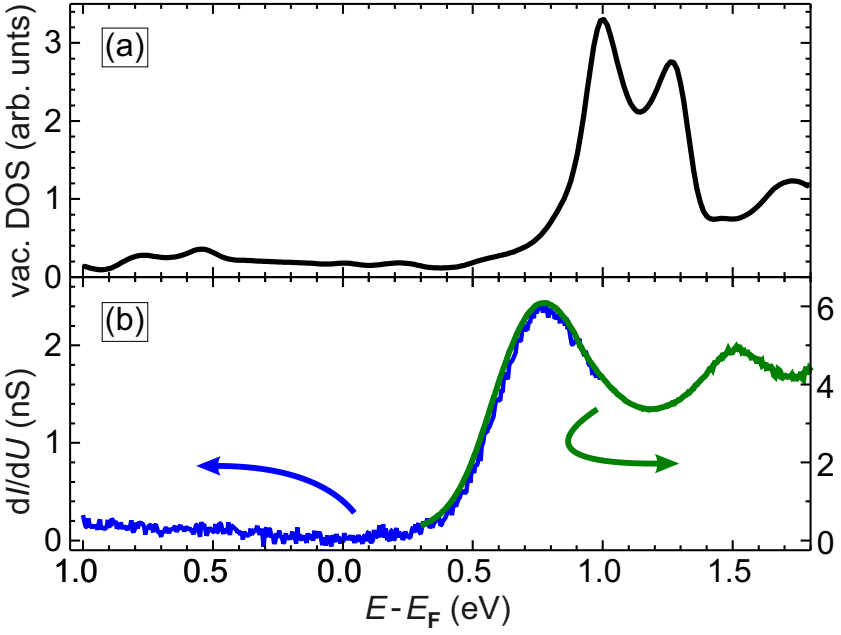

FIG. 3. (a) Vacuum LDOS $3 \AA$ above the Mn double layer on $\mathrm{W}(001)$ in the $c(2 \times 2)$ AFM state as calculated via DFT. (b) Tunneling differential conductance spectrum measured on the Mn double layer on $\mathrm{W}(001)$. Stabilization parameters: $U=1 \mathrm{~V}, I=1 \mathrm{nA}$ (blue); $U=0.3 \mathrm{~V}, I=0.5 \mathrm{nA}$ (green).

Majority- and minority-spin LDOS become indistinguishable in the $c(2 \times 2) \mathrm{AFM}$ state, resulting in a vanishing magnetic moment. Such behavior is expected for a transition metal which is only spin polarized by the adjacent magnetic layer. Since each interfacial $\mathrm{Mn}$ atom has four nearest neighbors in the surface layer, the $c(2 \times 2) \mathrm{AFM}$ order results in geometrical frustration. In contrast to the AFM Néel structure observed for the high-spin state of a ML Mn/ $\operatorname{Ag}(111)$ [43], however, frustration for strongly hybridized $\mathrm{Mn}$ in a low-spin state now results in the complete quenching of the magnetic moment.

Further confirmation for the correct description of the magnetic structure of the Mn DL on W(001) can be obtained from a thorough comparison of the spin-averaged electronic structure with experimental data. Using scanning tunneling spectroscopy we probed the local DOS in the vicinity of the Fermi energy by measuring the differential conductance $d I / d U$. Within the Tersoff-Hamann model [44] of STM the $d I / d U$ spectrum is directly proportional to the vacuum LDOS a few $\AA$ above the surface. We find a reasonable agreement between the calculated vacuum LDOS of the Mn DL on W(001) in the $c(2 \times 2)$ AFM state [Fig. 3(a)] and the measured $d I / d U$ signal [Fig. 3(b)]. In particular, the absence of features below and around the Fermi level and two peaks in the unoccupied states are reasonably well reproduced [45]. To investigate whether a $c(2 \times 2) \mathrm{AFM}$ with a finite magnetic moment can be stabilized in the Mn interface layer at increased distance between the Mn DL and the W(001) surface, i.e., by reducing the hybridization, we investigated the electronic properties of these hypothetical structures. However, our calculations reveal qualitative changes in the vacuum LDOS which are no longer in agreement with the experimental data (see Ref. [36] for details).

Even stronger evidence for the proposed magnetic ground state of the Mn DL is obtained by a comparison of SPSTM images with those calculated within the spin-polarized 


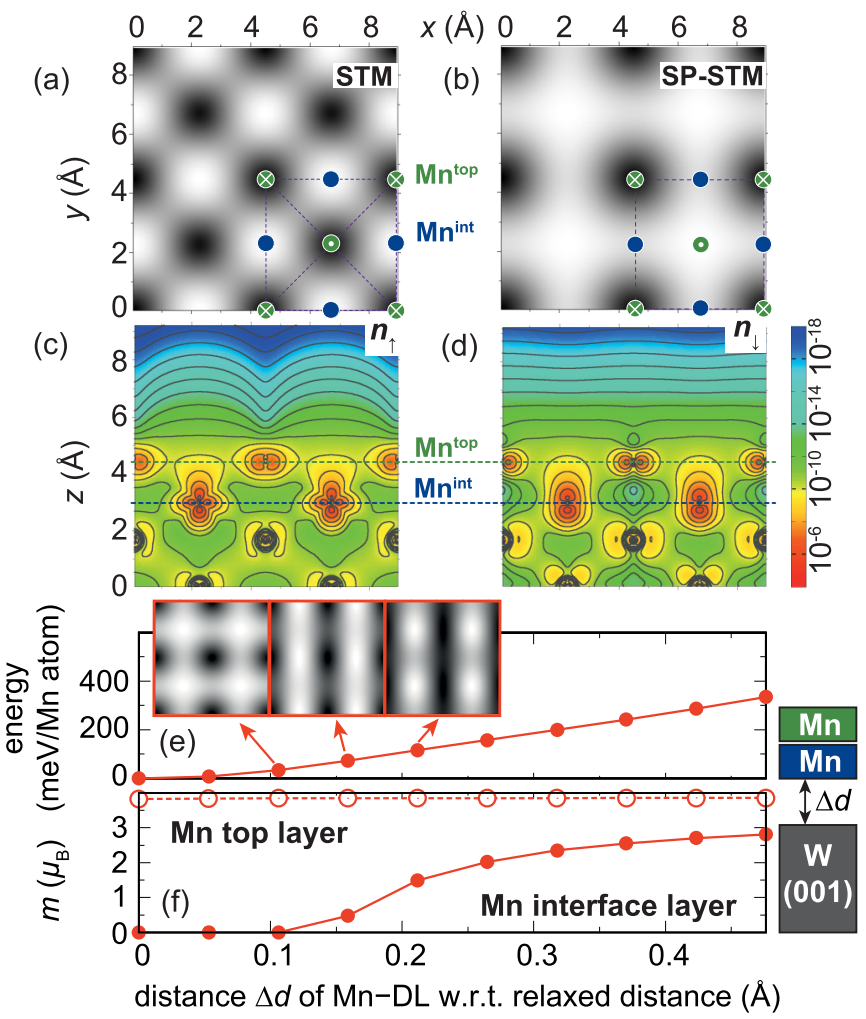

FIG. 4. (a) Calculated STM image $(U=+10 \mathrm{mV})$ of the $c(2 \times 2)$ AFM Mn DL on W(001) at a distance of $3 \AA$ above the surface. Surface and interface $\mathrm{Mn}$ atoms are denoted by green and blue solid circles, respectively. White crosses and dots denote the downwards and upwards pointing magnetic moments. Note that we find an outof-plane easy magnetization direction from DFT [36]. (b) Calculated SP-STM image as in (a) assuming a tip spin polarization of 0.5. (c), (d) Cross-section plots through the spin-up and spin-down partial charge density in the energy range $\left[E_{\mathrm{F}}, E_{\mathrm{F}}+0.01 \mathrm{eV}\right]$ for $y=0 \AA$ of (a). (e) Total energy and (f) magnetic moment of the Mn interface and surface layer in dependence of the distance between the Mn DL and the $\mathrm{W}(001)$ substrate with respect to the structurally relaxed distance. The insets show simulated SP-STM images for the three interlayer distances marked in (e).

version of the Tersoff-Hamann model [46]. Figure 4(a) shows a calculated STM image based on DFT for the $c(2 \times 2)$ AFM state. A small energy range above the Fermi energy has been considered. As expected, the STM image displays the chemical $p(1 \times 1)$ unit cell of the surface. However, the maxima are not in registry with surface $\mathrm{Mn}$ atoms but with interface Mn atoms. Calculated SP-STM images [Fig. 4(b)] show the magnetic $c(2 \times 2)$ unit cell with maxima and minima at the Mn surface atoms with opposite magnetic moments. A characteristic feature of the SP-STM image is that the contrast is also high between the Mn surface atoms, i.e., at the positions of the interface $\mathrm{Mn}$ atoms, which is due to the anticorrugation effect already observed in Fig. 4(a). This leads to a meshlike appearance of the SP-STM image which is in good agreement with experimental observations [cf. Fig. 1(c)]. Note that this effect has not been observed on the $\mathrm{Fe} \mathrm{ML}$ on $\mathrm{W}(001)$ which also possesses a $c(2 \times 2) \mathrm{AFM}$ ground state [37].
We can understand the unusual STM and SP-STM images based on the cross-sectional plots [Figs. 4(c) and 4(d)] through the spin-resolved integrated LDOS which leads to the images shown in Figs. 4(a) and 4(b). At the Mn interface layer we observe a $d_{z^{2}}$-like state in both the majority- and minority-spin channels. However, due to the spin-polarized states of the Mn surface layer these states exhibit a different penetration into the vacuum. In the majority-spin channel the $d_{z^{2}}$ state leads to a large vacuum LDOS above the interface Mn layer. In the minority-spin channel it is screened by the surface states. Note that the LDOS of the Mn surface layer is quite low in the vicinity of the Fermi energy which is probed by STM due to the large exchange splitting [cf. Fig. 2(a)].

By artificially increasing the distance between the Mn $\mathrm{DL}$ and the $\mathrm{W}(001)$ substrate by $\Delta d$ in our calculations we can reduce the hybridization at the $\mathrm{Mn}-\mathrm{W}$ interface. Up to $\Delta d=0.1 \AA$, the magnetic moment of $\mathrm{Mn}(\mathrm{I})$ is fully quenched [Fig. 4(f)]. At larger distances, a magnetic moment develops which rises up to $\approx 3 \mu_{\mathrm{B}}$ at $0.55 \AA$, whereas the magnetic moment of the Mn top layer remains constant. While the formation of a magnetic moment typically leads to an energy gain, here the total energy still increases monotonically upon lifting the Mn DL from the W(001) substrate [cf. Fig. 4(e)] due to reduced hybridization at the interface.

The insets show simulated SP-STM images at the onset of magnetic moment formation. These data reveal that we expected a striped SP-STM image if the Mn interface layer
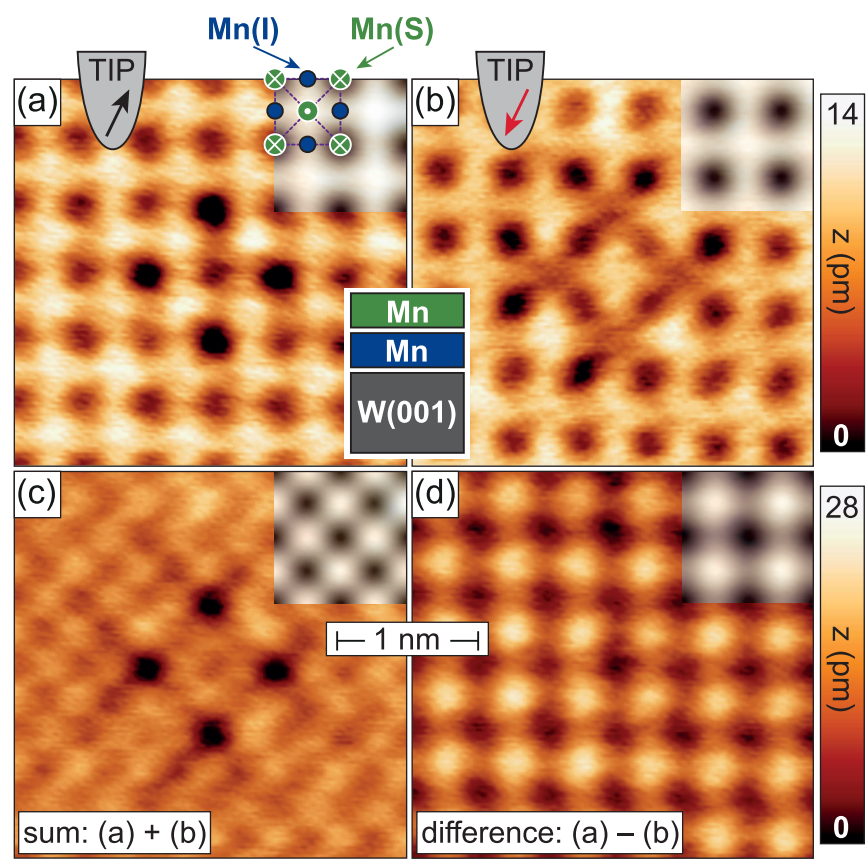

FIG. 5. (a), (b) SP-STM images of the Mn DL on W(001) with a tip magnetization which is reversed between the two images due to an uncontrolled tip switching event. Scan parameters: $U=$ $10 \mathrm{mV}, I=3 \mathrm{nA}$. The insets in the upper right corner show the calculated SP-STM image with a tip spin polarization of +0.5 as in Fig. 4(b) and with a reversed tip spin polarization of -0.5 . (c) Sum and (d) difference of the images of (a) and (b). Note that the defect structure in the center of the experimental image serves as a marker and that its origin is nonmagnetic. 
carried any magnetic moment. This is in stark contradiction to the experimental observation [Fig. 1(c)]. Therefore, the AFM order in the Mn top layer is only consistent with a complete quenching of the magnetic moment of the Mn interface layer.

Since the spin polarization is usually far below $100 \%$, SP-STM images such as the one presented in Fig. 1(c) result from the sum of spin-polarized and non-spin-polarized contributions to the tunneling current, representing the magnetic and spin-averaged electronic structure, respectively. As shown previously for the Fe ML on W(001) [37], they can be disentangled by imaging the same sample locations with opposite tip magnetization directions. Such a data set with a contrast reversal observed on the Mn DL on W(001) is presented in Figs. 5(a) and 5(b). Note the excellent agreement with the corresponding calculated SP-STM images (see insets). A defect located in the center of the experimental images can serve as a marker to align the two SP-STM images. Thereby, we can calculate the sum [Fig. 5(c)] and the difference image [Fig. 5(d)] which correspond to the non-spin-polarized and the spin-polarized part of the tunneling current, respectively. As expected, the electronic contrast due to the atomic lattice [Fig. 5(c)] is rotated by $45^{\circ}$ with respect to the magnetic unit cell [Fig. 5(d)]. A comparison of the observed electronic and magnetic contrast suggests that the bright spots correspond to the sites of the dead Mn interface layer shining through the surface layer. Such an STM observation of a subsurface layer has been previously reported for a nonmagnetic metal surface [34]. The magnetic contrast [Fig. 5(d)], on the other hand, looks very similar to that observed for the antiferromagnetic Fe ML on W(001) [37]. We would like to point out that the origin of the defect structure is nonmagnetic since it is absent in Fig. 5(d).

Conclusion. In conclusion, we have unraveled the magnetic ground state of the Mn DL on W(001). We find a $c(2 \times 2)$ AFM ground state for the surface Mn layer, whereas the Mn interface atoms carry no magnetic moment. The latter observation can be explained by a strong $\mathrm{Mn}-\mathrm{W}$ hybridization, resulting in a $\mathrm{Mn}$ interface layer which behaves more as a transition metal with a large spin susceptibility than one with an intrinsic magnetic moment. Our work shows the rich magnetic phases of Mn grown at the interface of transition metals.

Acknowledgments. M.B. acknowledges support of the experimental work by DFG (through SPP2137 "Skyrmionics," Grant No. BO-1468/26-1) and by the Dresden-Würzburg Center for Topological Quantum Matter Research (ct.qmat). We gratefully thank Gustav Bihlmayer for valuable discussions. S.M. and S.H. gratefully acknowledge computing time at the supercomputer of the North-German Supercomputing Alliance (HLRN). This project has received funding from the European Unions Horizon 2020 research and innovation programme under Grant Agreement No. 665095 (FET-Open project MAGicSky).
[1] T. Jungwirth, J. Sinova, A. Manchon, X. Marti, J. Wunderlich, and C. Felser, Nat. Phys. 14, 200 (2018).

[2] J. Barker and O. A. Tretiakov, Phys. Rev. Lett. 116, 147203 (2016).

[3] X. Zhang, Y. Zhou, and M. Ezawa, Sci. Rep. 6, 24795 (2016).

[4] T. Jungwirth, X. Marti, P. Wadley, and J. Wunderlich, Nat. Nanotechnol. 11, 231 (2016).

[5] L. Šmejkal, Y. Mokrousov, B. Yan, and A. H. MacDonald, Nat. Phys. 14, 242 (2018).

[6] P. Wadley, B. Howells, J. Železný, C. Andrews, V. Hills, R. P. Campion, V. Novák, K. Olejník, F. Maccherozzi, S. S. Dhesi, S. Y. Martin, T. Wagner, J. Wunderlich, F. Freimuth, Y. Mokrousov, J. Kuneš, J. S. Chauhan, M. J. Grzybowski, A. W. Rushforth, K. W. Edmonds et al., Science 351, 587 (2016).

[7] O. Gomonay, V. Baltz, A. Brataas, and Y. Tserkovnyak, Nat. Phys. 14, 213 (2018).

[8] D. Hobbs, J. Hafner, and D. Spišák, Phys. Rev. B 68, 014407 (2003).

[9] M. Bode, M. Hennefarth, D. Haude, M. Getzlaff, and R. Wiesendanger, Surf. Sci. 432, 8 (1999).

[10] D. A. Tulchinsky, J. Unguris, and R. J. Celotta, J. Magn. Magn. Mater. 212, 91 (2000).

[11] M. Bode, S. Heinze, A. Kubetzka, O. Pietzsch, M. Hennefarth, M. Getzlaff, R. Wiesendanger, X. Nie, G. Bihlmayer, and S. Blügel, Phys. Rev. B 66, 014425 (2002).

[12] T. K. Yamada, M. M. J. Bischoff, T. Mizoguchi, and H. van Kempen, Surf. Sci. 516, 179 (2002).

[13] J. Hafner and D. Spišák, Phys. Rev. B 72, 144420 (2005).

[14] J. T. Kohlhepp and W. J. M. de Jonge, Phys. Rev. Lett. 96, 237201 (2006).
[15] P.-J. Hsu, C.-I Lu, Y.-H. Chu, B.-Y. Wang, C.-B. Wu, L.-J. Chen, S.-S. Wong, and M.-T. Lin, Phys. Rev. B 85, 174434 (2012).

[16] J. L. Fry, Y. Z. Zhao, N. E. Brener, G. Fuster, and J. Callaway, Phys. Rev. B 36, 868 (1987).

[17] G. Fuster, N. E. Brener, J. Callaway, J. L. Fry, Y. Z. Zhao, and D. A. Papaconstantopoulos, Phys. Rev. B 38, 423 (1988).

[18] V. L. Moruzzi, Phys. Rev. Lett. 57, 2211 (1986).

[19] V. L. Moruzzi, P. M. Marcus, and P. C. Pattnaik, Phys. Rev. B 37, 8003 (1988).

[20] V. L. Moruzzi and P. M. Marcus, Phys. Rev. B 38, 1613 (1988).

[21] S. Heinze, M. Bode, A. Kubetzka, O. Pietzsch, X. Nie, S. Blügel, and R. Wiesendanger, Science 288, 1805 (2000).

[22] M. Bode, M. Heide, K. von Bergmann, P. Ferriani, S. Heinze, G. Bihlmayer, A. Kubetzka, O. Pietzsch, S. Blügel, and R. Wiesendanger, Nature (London) 447, 190 (2007).

[23] Y. Yoshida, S. Schröder, P. Ferriani, D. Serrate, A. Kubetzka, K. von Bergmann, S. Heinze, and R. Wiesendanger, Phys. Rev. Lett. 108, 087205 (2012).

[24] P. Ferriani, K. von Bergmann, E. Y. Vedmedenko, S. Heinze, M. Bode, M. Heide, G. Bihlmayer, S. Blügel, and R. Wiesendanger, Phys. Rev. Lett. 101, 027201 (2008).

[25] S. Dennler and J. Hafner, Phys. Rev. B 72, 214413 (2005).

[26] L. N. Liebermann, D. R. Fredkin, and H. B. Shore, Phys. Rev. Lett. 22, 539 (1969).

[27] L. Liebermann, J. Clinton, D. M. Edwards, and J. Mathon, Phys. Rev. Lett. 25, 232 (1970).

[28] C. Liu and S. D. Bader, Phys. Rev. B 41, 553 (1990).

[29] C. A. F. Vaz, J. A. C. Bland, and G. Lauhoff, Rep. Prog. Phys. 71, 056501 (2008). 
[30] S. Andrieu, M. Piecuch, and J. F. Bobo, Phys. Rev. B 46, 4909 (1992).

[31] D. Knab and C. Koenig, Phys. Rev. B 43, 8370 (1991).

[32] U. Gradmann, Appl. Phys. 3, 161 (1974).

[33] R. Wu and A. J. Freeman, Phys. Rev. B 44, 4449 (1991).

[34] S. Heinze, R. Abt, S. Blügel, G. Gilarowski, and H. Niehus, Phys. Rev. Lett. 83, 4808 (1999).

[35] A. Weismann, M. Wenderoth, S. Lounis, P. Zahn, N. Quaas, R. G. Ulbrich, P. H. Dederichs, and S. Blügel, Science 323, 1190 (2009).

[36] See Supplemental Material at http://link.aps.org/supplemental/ 10.1103/PhysRevResearch.2.012075 for detailed information regarding experimental and computational details, i.e., SP-STM contrast changes due to tip switching events and STM simulations.

[37] A. Kubetzka, P. Ferriani, M. Bode, S. Heinze, G. Bihlmayer, K. von Bergmann, O. Pietzsch, S. Blügel, and R. Wiesendanger, Phys. Rev. Lett. 94, 087204 (2005).

[38] www.flapw.de.
[39] J. P. Perdew, K. Burke, and M. Ernzerhof, Phys. Rev. Lett. 77, 3865 (1996).

[40] R. Yu, D. Singh, and H. Krakauer, Phys. Rev. B 43, 6411 (1991).

[41] Our DFT calculations on FM and LAFM states are consistent with those reported in Ref. [25].

[42] Interestingly, the relaxed interlayer distance between the Mn interface and the $\mathrm{W}$ surface layer is quite similar to that reported for an Fe ML on W(001) which showed an AFM ground state due to a strong Fe-W hybridization [37].

[43] C. L. Gao, W. Wulfhekel, and J. Kirschner, Phys. Rev. Lett. 101, 267205 (2008).

[44] J. Tersoff and D. R. Hamann, Phys. Rev. B 31, 805 (1985).

[45] We attribute the deviations between the experimental and theoretical peaks to the fact that the exchangecorrelation functional is only an approximation to the self-energy.

[46] D. Wortmann, S. Heinze, P. Kurz, G. Bihlmayer, and S. Blügel, Phys. Rev. Lett. 86, 4132 (2001). 